\title{
Nanoscale
}

Check for updates

Cite this: Nanoscale, 2021, 13, 2026

\section{Quantitative mapping of magnetic properties at the nanoscale with bimodal AFM $\dagger$}

\author{
Victor G. Gisbert, (ID a Carlos A. Amo, ${ }^{a}$ Miriam Jaafar, (iD b Agustina Asenjo (iD a and \\ Ricardo Garcia iD *a
}

Received 7th December 2020, Accepted 12th January 2021

DOI: $10.1039 /$ dOnr08662b

rsc.li/nanoscale

\begin{abstract}
We demonstrate that a force microscope operated in a bimodal configuration enables the mapping of magnetic interactions with high quantitative accuracy and high-spatial resolution ( $30 \mathrm{~nm})$. Bimodal AFM operation doubles the number of observables with respect to conventional magnetic force microscopy methods which enables to determine quantitatively in a single processing step several magnetic properties. The theory of bimodal AFM provides analytical expressions for different magnetic force models, in particular those characterized by power-law and exponential distance dependences. Bimodal AFM provides a self-evaluation protocol to test the accuracy of the measurements. The agreement obtained between the experiments and theory for two different magnetic samples support the application of bimodal AFM to map quantitatively long-range magnetic interactions.
\end{abstract}

\section{Introduction}

The emergence of hybrid materials that integrate different types of functionalities such as mechanical, electrical, magnetic, chemical or biological requires the development of quantitative, high-spatial resolution and efficient methods to map quantitatively spatial variations in the functionality or functionalities of interest. Atomic force microscopy has provided a variety of methods to map interfacial properties, ${ }^{1-8}$ among them, bimodal AFM offers a flexible platform for fast, quantitative and high-spatial resolution mapping of surfaces. ${ }^{9-11}$

Bimodal AFM is based on the simultaneous excitation and detection of two cantilever modes, commonly, the first and the second modes (Fig. 1a). General descriptions about bimodal AFM operation might be found elsewhere. ${ }^{8,10,11}$ The first bimodal AFM experiments exploited the observables associated with the 2nd mode to enhance compositional contrast. $^{12-18}$ A theoretical framework based on decomposing the equation of motion of the whole cantilever into the equations of motion of the excited modes ${ }^{19}$ enabled to transform the observables into mechanical properties such as the elastic modulus ${ }^{20,21}$ or viscoelastic coefficients. ${ }^{22,23}$ Thus,

\footnotetext{
${ }^{a}$ Instituto de Ciencia de Materiales de Madrid, CSIC, c/Sor Juana Inés de la Cruz 3, 28049 Madrid, Spain

${ }^{b}$ Departamento de Física de la Materia Condensada and Condensed Matter Physics Center (IFIMAC), Universidad Autónoma de Madrid, 28049 Madrid, Spain.

E-mail:r.garcia@csic.es

$\dagger$ Electronic supplementary information (ESI) available: Data to illustrate the self-evaluation protocol. See DOI: 10.1039/d0nr08662b
}
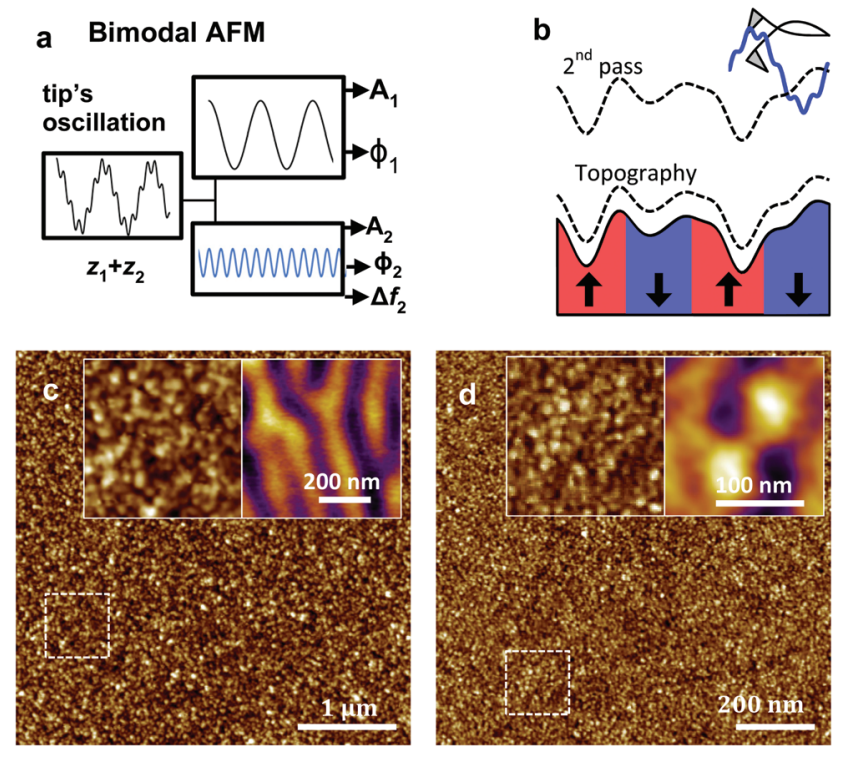

Fig. 1 (a) Tip's oscillation in bimodal AFM. The cantilever is excited at its first two eigenmodes. Upon interaction with the sample, the components of the tip's response are processed. This step generates several observables. (b) Second pass scheme applied in bimodal AFM for imaging magnetic interactions. $1^{\text {st }}$ pass: the height profile of the surface is obtained over a single scan line; $2^{\text {nd }}$ pass, the tip is lifted to a certain distance and displaced along the same line by following the recorded height profile. The bimodal observables are influenced by the magnetic interaction. The process is repeated over all scan lines (c) topographic image of the FePd film. The inset shows a high spatial resolution image of the topographic and the bimodal magnetic signal map $\left(\Delta f_{2}\right)$ of the same region. (d) (c) Topographic image of the HDD. The inset shows a high resolution image of the topographic and the bimodal magnetic signal map $\left(\phi_{2}\right)$ of the same region. 
bimodal AFM provided high-spatial resolution and accurate nanomechanical property maps of a large variety of materials and interfaces such as proteins, ${ }^{11,21,24,25} \mathrm{DNA}^{26-28}$ cells, ${ }^{29,30}$ bone microconstituents, ${ }^{31}$ lipid bilayers, ${ }^{32,33}$ liposomes loaded with nanoparticles, ${ }^{34}$ self-assembled monolayers, ${ }^{35}$ virus, ${ }^{36}{ }_{2 D}$ materials, ${ }^{37}$ organic crystals, ${ }^{38,39}$ solid-liquid interfaces ${ }^{40}$ and a variety of polymer surfaces. ${ }^{22,23,41,42}$

Bimodal AFM was also applied to generate material contrast images on heterogeneous samples made of regions with different electrical ${ }^{43-49}$ or magnetic properties. ${ }^{50-54}$ However, in those experiments and in particular in magnetic samples, it was not possible to determine the magnetic moment or the magnetic field of the material. The protocol and the theory to transform the bimodal observables under the interaction of a long-range electromagnetic force was not available.

Magnetic force microscopy (MFM) has been extensively applied to image magnetic nanostructures. ${ }^{6,55-58}$ The technique has experienced significant advances since the first implementations. ${ }^{59,60}$ However, the capability to combine quantitative information and high spatial resolution maps about magnetic properties remains very challenging. ${ }^{6}$

Here we demonstrate that bimodal AFM provides highspatial resolution quantitative maps of magnetic properties. The magnetic properties of a high-density disk and a FePd film were determined with a spatial resolution of about $30 \mathrm{~nm}$. Bimodal AFM provides also a self-evaluation protocol to determine the accuracy of the magnetic property maps.

The first section of the paper is devoted to introduce the theory that enables to transform the bimodal observables into magnetic properties. The generality of the theory is illustrated by proving analytical expressions for power-law and exponential magnetic interactions. The second section illustrates the capabilities of bimodal AFM to map a variety of properties such as the magnetic moment or the magnetic field. High spatial resolution $(\sim 30 \mathrm{~nm})$ maps of the magnetic properties of a hard disk drive and a FePd thin film are presented. The third section shows a self-evaluation criterion coming directly from the bimodal data to determine the accuracy of the measurement.

\section{Theory of bimodal AFM for long- range magnetic interactions}

The analytical expressions that relate the magnetic parameters of a material to bimodal AFM observables are deduced by recording the changes of the amplitude $A_{1}$ and phase shift $\phi_{1}$ of the first mode and the amplitude and either the phase shift $\phi_{2}$ or frequency shift $\Delta f_{2}$ of the $2^{\text {nd }}$ mode (Fig. 1a). The frequency shift $\Delta f_{2}$ represents the shift in the resonant frequency of the second mode with respect to its free (absence of tipsample interaction forces) resonant frequency $f_{02}$. The measurements were performed with a two-pass approach (Fig. 1b), therefore, feedback controls were not applied during the recording of the observables.
To relate the bimodal AFM observables with the magnetic parameters of a sample requires (i) a model of the tip-sample magnetic interaction and (ii) to solve the virial equations for the first and second mode of the cantilever. We assume that the tip-sample force dependence on the distance is known. In particular, we present the theory for two different magnetic models, a dipole (tip)-dipole (sample) model and a tip-sample transfer function model. ${ }^{55,56}$

For a dipole-dipole interaction model we assume that the dipoles are oriented perpendicular to the sample surface (Fig. 1b), then the force acting on a magnetized tip is approximated by ${ }^{57}$

$$
F_{\mathrm{z}}(x, y, z)=m_{\mathrm{tip}} \frac{\delta B_{\mathrm{z}}}{\delta z}=\frac{3}{2 \pi} \frac{\mu_{0} m_{\mathrm{tip}} m_{\mathrm{s}}(x, y, z)}{z_{\mathrm{c}}{ }^{4}}
$$

where $B_{\mathrm{z}}, m_{\mathrm{tip}}$, and $m_{\mathrm{s}}$ are, respectively, the component of the magnetic field in the direction perpendicular to the sample, the magnetic moment of the tip and the sample; $\mu_{0}$ the permittivity of the vacuum, $z_{\mathrm{c}}$ the average tip-sample distance.

For a tip-sample magnetic interaction deduced by using the tip transfer-function theory ${ }^{55,56}$

$$
\begin{gathered}
F_{\mathrm{z}}(x, y, z)=\boldsymbol{m} \cdot \nabla \boldsymbol{B}=-\mu_{0} m_{\mathrm{tip}} \kappa e^{-\kappa z} \frac{\left(1-e^{-\kappa t}\right)}{2} M(\boldsymbol{k}) \\
=-m_{\mathrm{tip}} \kappa B_{0} e^{-\kappa z}=F_{0} e^{-\kappa z}
\end{gathered}
$$

where $M(\boldsymbol{k})$ is the Fourier transform of the sample magnetization $M_{\mathrm{z}}(\boldsymbol{r}){ }^{55,56}$ The vectors $\boldsymbol{k}$ and $\boldsymbol{r}$ satisfy.

$$
|\boldsymbol{k}|=\kappa=\sqrt{k_{x}^{2}+k_{y}^{2}} \text { and }|\boldsymbol{r}|=\sqrt{x^{2}+y^{2}} .
$$

The second step in the development of the theory involved to apply the virial theorem to the tip's motion. ${ }^{61} \mathrm{We}$ approximated the motion of the cantilever-tip system by the elastic beam equation of a rectangular cantilever ${ }^{62}$ and subsequently we projected the motion into the components of the excited modes by ref. ${ }^{63}$

$F_{\mathrm{ts}}(t)+F_{1} \cos \left(2 \pi f_{1} t\right)=k_{1} /\left(2 \pi f_{1}\right)^{2} \ddot{z}_{1}+\left(k_{1} /\left(Q_{1} 2 \pi f_{01}\right)\right) \underline{z}_{1}+k_{1} z_{1}$

$F_{\text {ts }}(t)+F_{2} \cos \left(2 \pi f_{2} t\right)=k_{2} /\left(2 \pi f_{2}\right)^{2} \ddot{z}_{2}+\left(k_{2} /\left(Q_{2} 2 \pi f_{02}\right)\right) z_{2}+k_{2} z_{2}$

where $f_{i}, f_{0 i}, Q_{i}, k_{i}$ and $F_{i}$ are, respectively, the driving frequency, the free resonant frequency, the quality factor, the stiffness and the driving force of the $i$-th mode. $F_{\mathrm{ts}}$ is the tipsample interaction force. The vertical motion of the tip (deflection) is decomposed in two components oscillating at the resonant frequencies,

$$
\begin{aligned}
z(t) & =z_{0}+z_{1}(t)+z_{2}(t) \\
& \approx A_{1} \cos \left(2 \pi f_{1} t-\phi_{1}\right)+A_{2} \cos \left(2 \pi f_{2} t-\phi_{2}\right)
\end{aligned}
$$

where $z_{0}, z_{1}$ and $z_{2}$ are, respectively, the static, the first and the second mode deflections; $A_{1}$ and $A_{2}$ are the oscillation amplitudes and $\phi_{1}$ and $\phi_{2}$ are, respectively, the phase shifts of the first and second mode. 
The amplitude of the first mode $A_{1}$ is set to a fixed value $A_{\mathrm{sp}}$ (set-point amplitude) which is lower than the free amplitude $A_{01}$ during the measurement of the topography. To facilitate the deduction of analytical expressions, we assume that the value of $A_{2}$ is much smaller than the value of $A_{1}$ and $z_{0}$ is negligible with respect to both $A_{1}$ and $A_{2}$.

To relate the observables with the tip-sample force we applied the virial $V_{i}$ equations ${ }^{19,21}$ to the excited modes

$$
\begin{gathered}
V_{1}=f_{1} \int_{0}^{1 / f_{1}} F_{\mathrm{ts}}(z(t)) z_{1}(t) \mathrm{d} t \\
V_{2}=f_{2} \int_{0}^{1 / f_{2}} F_{\mathrm{ts}}(z(t)) z_{2}(t) \mathrm{d} t \approx\left(A_{2}{ }^{2} / 4 \pi\right) \int_{0}^{1 / f_{1}} F^{\prime}(t) \mathrm{d} t .
\end{gathered}
$$

The above equations can be resolved in terms of the observables without knowing the explicit expression of the interaction force. This was accomplished by integrating the equation of motion of the modes over a period. ${ }^{19,21}$

For a bimodal AFM configuration characterized by a single amplitude modulation feedback loop acting on the $1^{\text {st }}$ mode, the virials $V_{1}$ and $V_{2}$ are determined by

$$
\begin{gathered}
V_{1}=-\left(\left(k_{1} A_{1} A_{01}\right) /\left(2 Q_{1}\right)\right) \cos \phi_{1} \\
V_{2}=-\left(\left(k_{2} A_{2} A_{02}\right) /\left(2 Q_{2}\right)\right) \cos \phi_{2} .
\end{gathered}
$$

For a bimodal AFM configuration that incorporates two feedback loops; an amplitude modulation feedback loop acting on the $1^{\text {st }}$ mode and a phase-locked loop to track the shift of the resonant frequency of the second mode $\left(\phi_{2}=\pi /\right.$ $2)^{64}, V_{2}$ is obtained by

$$
V_{2}=-k_{2} A_{2}^{2} \Delta f_{2} / f_{02} .
$$

By combining eqn (1), (9) and (11), the sample magnetic moment (dipole-dipole interaction) is given by

$$
m_{\mathrm{s}}=-\frac{2 \pi}{3 \mu_{0} m_{\mathrm{tip}}} V_{1} A_{1}\left[\left(\frac{z_{\mathrm{c}}}{A_{1}}\right)^{2}-1\right]^{3 / 2}
$$

and the magnetic field created by the sample dipoles at $z=z_{\mathrm{c}}$,

$$
B_{\mathrm{z}}=\frac{\mu_{0} m_{\mathrm{s}}}{2 \pi z_{\mathrm{c}}{ }^{3}} .
$$

The mean distance $z_{\mathrm{c}}$ might be determined from the bimodal AFM observables as

$$
z_{\mathrm{c}}=A_{1} \frac{\sqrt{2}}{4} \frac{\sqrt{3+12 \chi+\sqrt{120 \chi^{2}+80 \chi+25}}}{\sqrt{1-\chi}}
$$

with the intermediate variable $\chi$ given by

$$
\chi=\frac{V_{1} A_{2}^{2}}{V_{2} A_{1}^{2}}
$$

alternatively, $z_{\mathrm{c}}$ could be approximated by $z_{\mathrm{c}} \approx A_{\mathrm{sp}}+A_{2}+$ lift height.

For the tip transfer-function model (eqn (2)), the virials $V_{i}$ are given by

$$
\begin{aligned}
& V_{1}\left(z_{\mathrm{c}}\right)=-F_{0} e^{-\kappa z_{\mathrm{c}}} A_{1} I_{1}\left(\kappa A_{1}\right) I_{0}\left(\kappa A_{2}\right) \\
& V_{2}\left(z_{\mathrm{c}}\right)=-F_{0} e^{-\kappa z_{\mathrm{c}}} A_{2} I_{1}\left(\kappa A_{2}\right) I_{0}\left(\kappa A_{1}\right)
\end{aligned}
$$

where $I_{0}$ and $I_{1}$ are the modified Bessel functions of first kind. The magnetic wave number $\kappa$ was determined by using eqn (9) and (10)

$$
\kappa=2 \sqrt{2} \sqrt{\frac{A_{1}{ }^{2} V_{2}-A_{2}{ }^{2} V_{1}}{A_{1}{ }^{4} V_{2}-2 A_{1}{ }^{2} A_{2}{ }^{2} V_{1}+2 A_{1}{ }^{2} A_{2}{ }^{2}-A_{2}{ }^{4} V_{1}}} .
$$

Once the magnetization wave number is known, the magnetic field at a given distance from the surface is obtained by

$$
B\left(z=z_{\mathrm{c}}\right)=\frac{1}{\kappa \cdot m_{\text {tip }}} \frac{V_{1}\left(z_{\mathrm{c}}\right)}{A_{1} I_{1}\left(\kappa A_{1}\right) I_{0}\left(\kappa A_{2}\right)}
$$

which on the sample surface gives

$$
B_{0}=\frac{e^{\kappa z_{\mathrm{c}}}}{\kappa \cdot m_{\mathrm{tip}}} \frac{V_{1}\left(z_{\mathrm{c}}\right)}{A_{1} I_{1}\left(\kappa A_{1}\right) I_{0}\left(\kappa A_{2}\right)} .
$$

\section{Materials and methods}

\subsection{Bimodal AFM set-up and magnetic property mapping}

We developed a custom-made code to process the bimodal AFM data. The code was incorporated into a commercial AFM (Cypher S, Asylum Research-Oxford Instruments). To separate the magnetic interactions from the topography of the samples, we applied a two-pass method ${ }^{65}$ (the so called lift-mode). In first pass the topography of the sample along a given line was obtained. Immediately afterwards, the tip was lifted some distance from the surface and displaced over the same line by following the topography profile ( $2^{\text {nd }}$ pass) (Fig. 1b). The lift height was about $2 \mathrm{~nm}$ on the HDD and $20 \mathrm{~nm}$ on the FePd film. Those values were chosen to maximize the magnetic contrast of the observables of the $2^{\text {nd }}$ mode.

In bimodal AFM, the first and second mode of the cantilever are excited and detected at the same time. The amplitudes of $A_{1}$ and $A_{2}$ used here were, respectively, in the 7-42 nm and 1-10 $\mathrm{nm}$ ranges (see figure captions for specific details). To detect frequency shifts associated with the $2^{\text {nd }}$ mode requires the use of a phase-locked loop to keep at $90^{\circ}$ the phase shift between the excitation signal and the cantilever-tip response.

The two-pass method did not suppress completely the influence of non-magnetic long-range interactions such as van der Waals or electrostatic forces. In fact, in our experiments those long-range interactions were comparable to the magnetic interaction. Several procedures have been developed in MFM to remove non-magnetic effects from the data. ${ }^{50,66,67}$ Here we developed a method to separate the magnetic force from nonmagnetic long-range forces especially suited for bimodal AFM operation. The method is based on the additive character of the forces and the virials. It has three main steps. 
First, in the case that the force acting on the tip has different origins, for example, $F_{\mathrm{m}}$ (magnetic) and $F_{\mathrm{nm}}$ (nonmagnetic), the virial of mode $i$ satisfies

$$
V_{i}=V_{i}^{m}+V_{i}^{n m} .
$$

Second, we proceed to determine the contribution of the non-magnetic forces in the virials. This was achieved by calculating the average value of the virial in the map,

$$
\begin{aligned}
\left\langle V_{i}\right\rangle=\frac{\sum_{m, n} V_{i}\left(x_{m}, y_{n}\right)}{M+N} & =\frac{\sum_{m, n} V_{i}^{n m}\left(x_{m}, y_{n}\right)}{M+N} \\
\left\langle V_{i}^{m}\right\rangle & =0 .
\end{aligned}
$$

Over a large region of the HDD, the virials of the up domains are cancelled by the virials of the down domains. The FePd film was measured on the demagnetized state, therefore the number of up regions coincide with the number of down regions.

Third, a new set of virial maps is generated by using

$$
V_{i}^{*}(x, y)=V_{i}(x, y)-\left\langle V_{i}\right\rangle .
$$

Finally, the new virials are introduced into the eqn (9), (10), (11), (16) and (17).

\subsection{Magnetic samples}

Two different magnetic samples were used in this work. A commercial high-density disk (HDD) from Toshiba (Japan) made of a CoCrPt alloy with perpendicular anisotropy. The HDD was pre-patterned with $50 \mathrm{~nm}$ bits. The other sample was a FePd thin film (40 $\mathrm{nm}$ ) grown by sputtering on a MgO substrate at room temperature. The FePd sample, demagnetized before the experiments, presented a stripe domain configuration. ${ }^{68}$ The magnetic moment of the stripes was aligned perpendicular to the surface. The comparison with the topographic images (Fig. 1c and d) illustrates the absence of any correlation between topography images and magnetic property maps.

\subsection{Cantilever type and calibration}

SSS-MFMR (Nanosensors, Germany) cantilevers with $f_{1}=75$ $\mathrm{kHz}, k_{1}=2.8 \mathrm{~N} \mathrm{~m}^{-1}, Q_{1}=160, f_{2}=400 \mathrm{kHz}, k_{2}=90 \mathrm{Nm}^{-1}, Q_{2}=$ 400 , were used to image the magnetic samples. The tip had a hard magnetic coating and a radius of curvature $<15 \mathrm{~nm}$. The coating is characterized by magnetization of $m_{\text {tip }} \approx 2.5 \times 10^{-14}$ emu and a coercivity $\approx 125$ Oe. Before performing the bimodal AFM experiments, the tip was magnetized by using of a strong permanent magnet.

Quantitative property mapping by bimodal AFM operation required to calibrate the force constant of the excited modes. ${ }^{69}$ The force constant of the first mode was calibrated using the multiple reference calibration method. ${ }^{70}$ This method avoided the mechanical contact between the tip and the sample during the calibration. The second mode of the cantilever was calibrated by using the stiffness-frequency power law relationship, $k_{2}=k_{1}\left(f_{2} / f_{1}\right)^{\zeta_{2}}$, where $\zeta_{2}$ is an experimental calibration parameter. $^{71}$

\subsection{Definition of material contrast}

The material contrast of a given observable was defined as

$$
\text { contrast }=\frac{1}{2} \frac{(\max (\operatorname{signal})-\min (\operatorname{signal}))}{\text { noise }} .
$$

The signal was measured as the peak-to-peak difference over two consecutive magnetic domains. The noise was measured by calculating the standard deviation on an area of the map that has a constant value of the magnetic field.
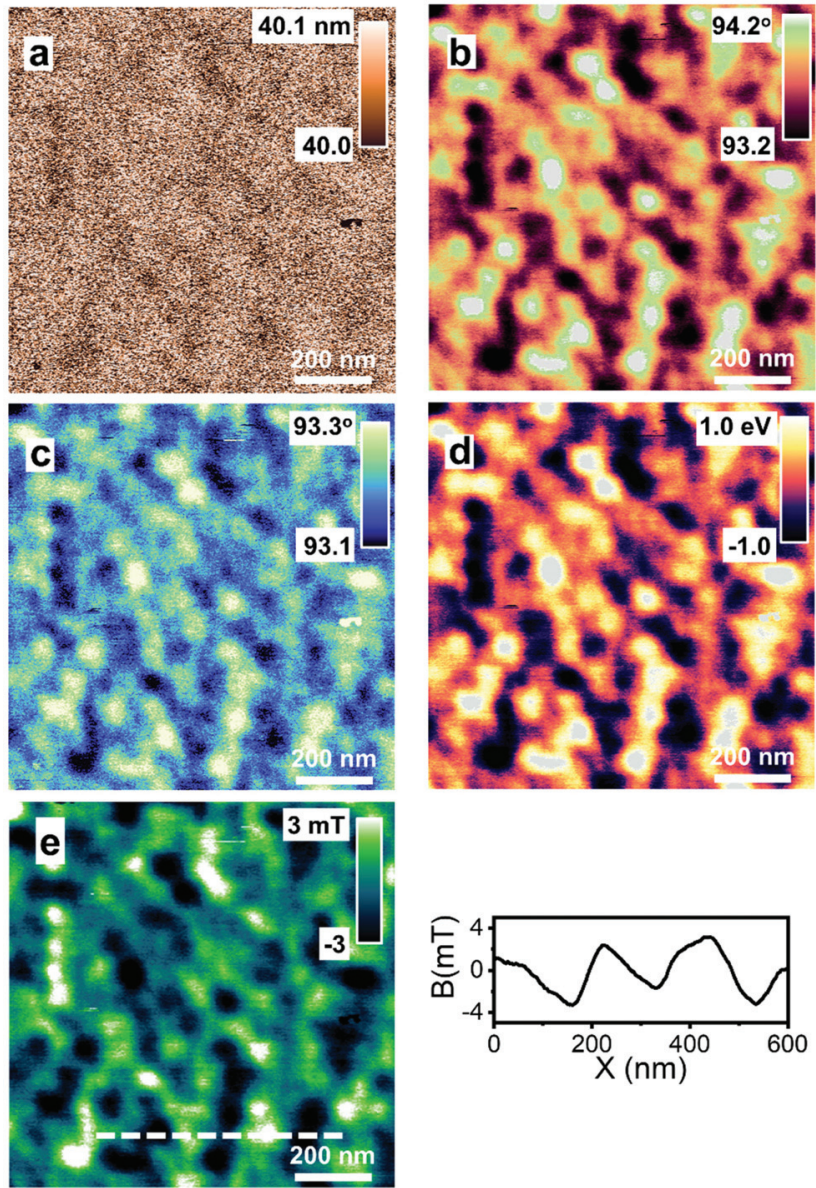

Fig. 2 Bimodal AFM maps of a HDD sample. (a) $A_{1}(x, y)$. (b) $\phi_{1}(x, y)$. (c) $\phi_{2}(x, y)$. (d) $V_{1}(x, y)$. (e) Magnetic field map probed at $45 \mathrm{~nm}$ from the surface. The map was obtained by processing $(a-d)$ maps. The magnetic interaction between the tip and the sample was described by the force transfer function model. The cross-section shows the variation of the field along the along dashed line marked on the map. Tip lift $=8 \mathrm{~nm}, z_{\mathrm{c}}$ $\approx A_{\text {sp }}+A_{2}+8 \mathrm{~nm}$; bimodal AFM parameters: $A_{01}=41 \mathrm{~nm}, A_{02}=10 \mathrm{~nm}$, $A_{1}=40 \mathrm{~nm} ; f_{1}=79 \mathrm{kHz}, f_{2}=493 \mathrm{kHz}, k_{1}=3.4 \mathrm{~N} \mathrm{~m}^{-1}, k_{2}=172 \mathrm{~N} \mathrm{~m}^{-1}, Q_{1}$ $=160, Q_{2}=502$. Scan line rate, $1 \mathrm{~Hz}$; maps of $512 \times 512$ pixels. 

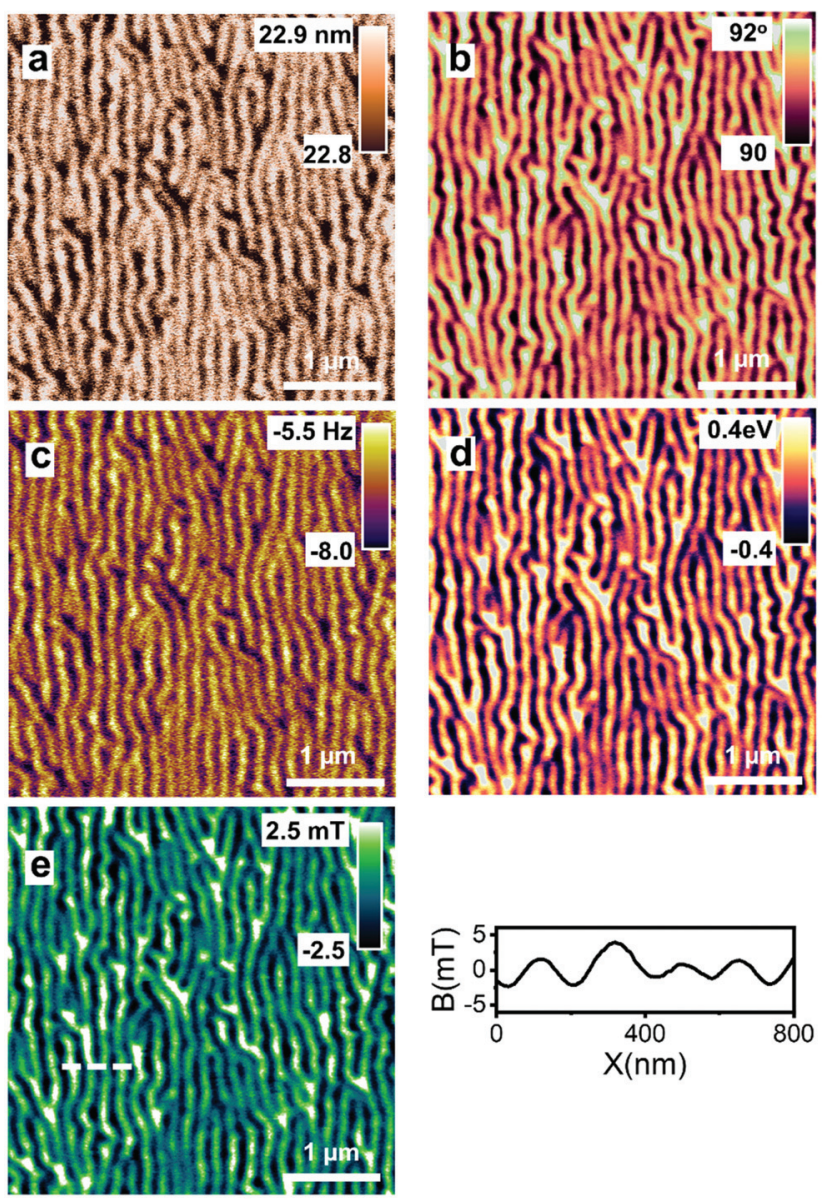

Fig. 3 Bimodal AFM maps of a FePd sample. (a) $A_{1}(x, y)$. (b) $\phi_{1}(x, y)$. (c) $\Delta f_{2}(x, y)$. (d) $V_{1}(x, y)$. (e) Magnetic field map probed at $35 \mathrm{~nm}$ from the surface. The map was obtained by processing (a-d) maps. Maps of $1024 \times$ 1024 pixels. The magnetic interaction between the tip and the sample was described by the force transfer function model. The cross-section shows the variation of the field along the along dashed line marked on the map. Tip lift $=20 \mathrm{~nm}, z_{\mathrm{c}} \approx A_{\mathrm{sp}}+A_{2}+20 \mathrm{~nm}$; bimodal AFM parameters: $A_{01}=$ $23.4 \mathrm{~nm}, A_{02}=5.0 \mathrm{~nm}, A_{1}=22.9 \mathrm{~nm} ; f_{1}=65 \mathrm{kHz}, f_{2}=412 \mathrm{kHz}, k_{1}=2.0 \mathrm{~N}$ $\mathrm{m}^{-1} ; k_{2}=102 \mathrm{~N} \mathrm{~m}^{-1}, Q_{1}=152, Q_{2}=396$. Scan line rate, $2 \mathrm{~Hz}$.

\section{Results and discussion}

The accuracy and the spatial resolution capabilities of bimodal AFM were tested on the HDD and the FePd film. A quick comparison between the AFM topographic and the magnetic images (Fig. 1c and d) revealed the absence of any correlation. To determine the magnetic properties of the sample required a model of the probe magnetization. Here, the tip is considered to have a net magnetic moment $m_{\text {tip }}$. The tip-sample magnetic interaction transfer function model proposed by Hug et al. is considered the most suitable theoretical framework to describe MFM experiments at the nanoscale. ${ }^{55,56}$ Therefore, the experiments are explained by using the theory of bimodal AFM for interactions characterized by an exponential dependence on the tip-sample distance. In this model, the force exerted by the sample's stray magnetic field in the perpendicular direction is determined in the Fourier space by introducing magnetization wave number $\mathbf{k}(x, y)$.

\subsection{Bimodal AFM mapping of magnetic properties}

Fig. 2a-d shows the maps of the $A_{1}, \phi_{1}, \phi_{2}$ and $V_{1}$ obtained on the HDD sample at $z_{\mathrm{c}}=45 \mathrm{~nm}$. The maps revealed the same magnetic domain structure. The magnetic contrast depended on the observable. The contrast for $A_{1}$ and $\phi_{1}$ was, respectively, 1.8 and 4.3 while $\Delta f_{2}$ and $V_{1}$ gave a contrast of 1.3 and 4.5. A map of the magnetic field is shown in Fig. 2e. The crosssection showed the variations of the magnetic field along the dashed line. It oscillated between -3.7 and $3.8 \mathrm{mT}$.

Fig. 3 shows the maps of the $A_{1}, \phi_{1}$ and $\Delta f_{2}$ and $V_{1}$ obtained on the FePd film at $z_{\mathrm{c}}=42 \mathrm{~nm}$. The maps revealed the longitudinal character of the magnetic domains. The contrast obtained in the $\phi_{1}(x, y)$ and $V_{1}(x, y)$ maps was, respectively, of 9.8; $V_{2}(x, y)$ and $\Delta f_{2}(x, y)$ have a contrast of 3 . However, the magnetic domain structure seems identical. The map of the stray magnetic field $\left(z_{\mathrm{c}}=42 \mathrm{~nm}\right)$ varied between -3 and $3 \mathrm{mT}$ (Fig. 3e). By changing the lift value, it is possible to determine the maps at other distances.
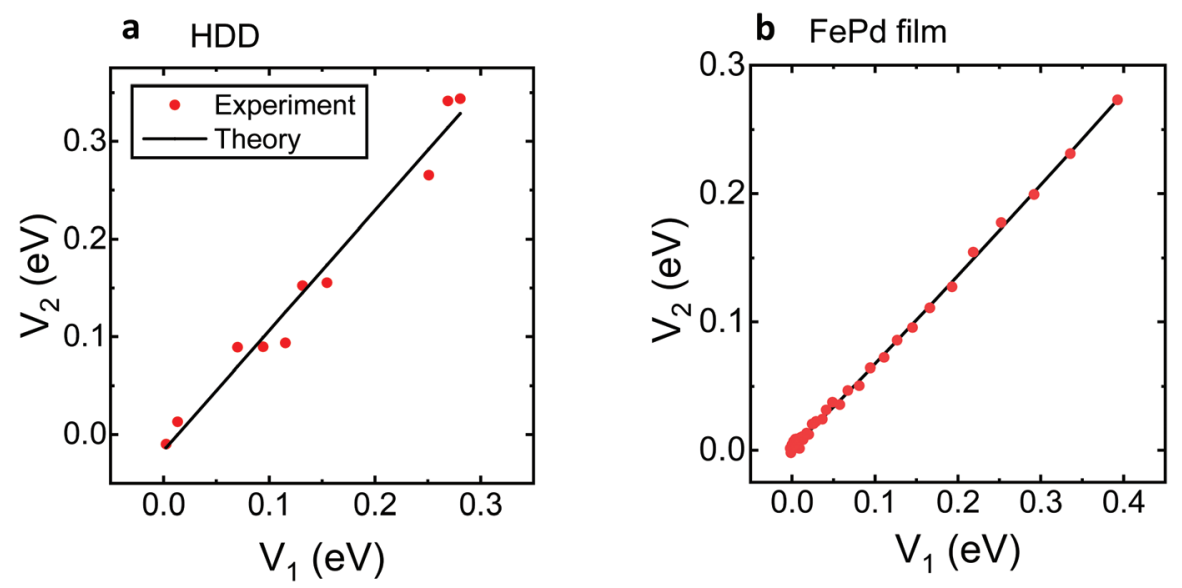

Fig. 4 Self-evaluation of the quantitative accuracy of bimodal AFM. (a) $V_{2}$ dependence on $V_{1}$ for the HDD sample. (b) $V_{2}$ dependence on $V_{1}$ for the FePd D sample. $V_{1}$ is modified by changing the lift height, respectively, from 10 to $150 \mathrm{~nm}$ for the HDD and 20 to $200 \mathrm{~nm}$ from the FePd film. The agreement obtained between theory and experiment confirms the accuracy of the numerical values provided by the bimodal AFM maps. 


\subsection{Self-evaluation criterion}

Bimodal AFM offers a self-evaluation criterion to verify whether the quantitative data obtained on the sample's magnetic properties do represent the real properties of the sample. The criterion comes from the bimodal AFM equations (see section 2). The theory shows the cross-representation of the virials $V_{1}$ and $V_{2}$ should follow a straight line if the magnetic interaction is dominated by an exponentially decaying force. Therefore, the accuracy of the magnetic field maps might be determined by plotting the experimental dependence of $V_{2}$ versus $V_{1}$. If the cross-representation of the virials departs significantly from a straight line, it should be concluded that the model applied in bimodal to transform the observables into magnetic properties was not appropriate.

Fig. 4 shows the cross-representations of the virials of the excited modes for the HDD and FePd film samples. Both samples, and in particular the FePd film, follow a straight line as predicted by the theory. These results underlined that the approximations used to develop the bimodal AFM theory are fully consistent with the experimental data.

\section{Conclusions}

Bimodal AFM has expanded the quantitative characterization of materials at the nanoscale by providing high resolution maps of magnetic parameters. Bimodal AFM generates quantitative $2 \mathrm{D}$ maps of the magnetic moment and the magnetic field created by a magnetized sample with high quantitative accuracy. We have developed the theory of bimodal AFM to transform the observables into magnetic parameters in terms of the tip transfer function model. This theory enables the mapping of the sample's magnetic properties at different tipsample distances. The accuracy and spatial resolution has been tested on a hard disk drive and FePd thin film samples. In both cases, the bimodal AFM observables provided highspatial resolution maps of the magnetic domain structure.

The bimodal AFM has two key features that are not found in any other magnetic force microscopy method. First, it is efficient. It just requires the recording of four data points per pixel to generate several magnetic property maps. Second, it provides a criterion to determine whether the measured magnetic property map gives a faithful characterization of the sample. To our best knowledge, this self-evaluation criterion is unique to bimodal AFM.

In summary, this contribution expands the quantitative characterization capabilities of bimodal force microscopy by providing a robust, reliable and high spatial resolution method to measure magnetic properties.

\section{Author contributions}

VGG performed the experiments and developed the bimodal AFM code. CAA developed the bimodal AFM theory. VGG and RG adapted the theory for magnetic property mapping and developed the self-evaluation criterion. AA and MJ provided the magnetic samples and performed additional macroscopic magnetic property measurements. RG conceived the project and wrote the manuscript. All the authors discussed the data and revised the manuscript.

\section{Conflicts of interest}

There are no conflicts of interest to declare.

\section{Acknowledgements}

Financial support from the Ministerio de Ciencia, Innovación y Universidades (PID2019-106801GB-I00; PID2019108075RB-C31; MAT2016-76507-R) and Comunidad de Madrid (SI1/PJI/2019-00055) are acknowledged.

\section{References}

1 R. Garcia and E. T. Herruzo, Nat. Nanotechnol., 2012, 7, 217-226.

2 S. Zhang, H. Aslan, F. Besenbacher and M. Dong, Chem. Soc. Rev., 2014, 43, 7412-7429.

3 Y. F. Dufrêne, T. Ando, R. Garcia, D. Alsteens, D. MartinezMartin, A. Engel, C. Gerber and D. J. Muller, Nat. Nanotechnol., 2017, 12, 295-307.

4 L. Collins, J. I. Kilpatrick, S. V. Kalinin and B. J. Rodriguez, Rep. Prog. Phys., 2018, 81, 086101.

5 D. Wang and T. P. Russell, Macromolecules, 2018, 51, 3-24.

6 O. Kazakova, R. Puttock, C. Barton, H. Corte-León, M. Jaafar, V. Neu and A. Asenjo, J. Appl. Phys., 2019, 125, 060901.

7 G. Stan and S. W. King, J. Vac. Sci. Technol., B: Nanotechnol. Microelectron.: Mater., Process., Meas., Phenom., 2020, 38, 060801.

8 R. Garcia, Chem. Soc. Rev., 2020, 49, 5850-5884.

9 T. R. Rodriguez and R. Garcia, Appl. Phys. Lett., 2004, 84, 449-451.

10 R. Garcia and R. Proksch, Eur. Polym. J., 2013, 49, 18971906.

11 S. Benaglia, V. G. Gisbert, A. P. Perrino, C. A. Amo and R. Garcia, Nat. Protoc., 2018, 13, 2890-2907.

12 R. Proksch, Appl. Phys. Lett., 2006, 89, 113121.

13 N. F. Martinez, S. Patil, J. R. Lozano and R. Garcia, Appl. Phys. Lett., 2006, 89, 153115.

14 S. Patil, N. F. Martinez, J. R. Lozano and R. Garcia, J. Mol. Recognit., 2007, 20, 516-523.

15 S. D. Solares and G. Chawla, Meas. Sci. Technol., 2010, 21, 125502.

16 D. Ebeling, B. Eslami and S. D. J. Solares, ACS Nano, 2013, 7, 10387-10396.

17 S. An, S. D. Solares, S. Santos and D. Ebeling, Nanotechnology, 2014, 25, 475701. 
18 S. Santos, C. Y. Lai, T. Olukan and M. Chiesa, Nanoscale, 2017, 9, 5038-5043.

19 J. R. Lozano and R. Garcia, Phys. Rev. B: Condens. Matter Mater. Phys., 2009, 79, 014110.

20 A. Labuda, M. Kocuń, W. Meinhold, D. Walters and R. Proksch, Beilstein J. Nanotechnol., 2016, 7, 970-982.

21 C. A. Amo, A. P. Perrino, A. F. Payam and R. Garcia, ACS Nano, 2017, 11, 8650-8659.

22 E. T. Herruzo, A. P. Perrino and R. Garcia, Nat. Commun., 2014, 5, 1-8.

23 S. Benaglia, C. A. Amo and R. Garcia, Nanoscale, 2019, 11, 15289-15297.

24 D. Martinez-Martin, E. T. Herruzo, C. Dietz, J. GomezHerrero and R. Garcia, Phys. Rev. Lett., 2011, 106, 198101.

25 G. Lamour, C. K. Yip, H. Li and J. Gsponer, ACS Nano, 2014, 8, 3851-3861.

26 C. Y. Lai, S. Santos and M. Chiesa, ACS Nano, 2016, 10, 6265-6272.

27 C.-Y. Y. Lai, S. Perri, S. Santos, R. Garcia and M. Chiesa, Nanoscale, 2016, 8, 9688-9694.

28 M. Kocun, A. Labuda, W. Meinhold, I. Revenko and R. Proksch, ACS Nano, 2017, 11, 10097-10105.

29 A. X. Cartagena-Rivera, W. H. Wang, R. L. Geahlen and A. Raman, Sci. Rep., 2015, 5, 11692.

30 D. Guan, E. Charlaix, R. Z. Qi and P. Tong, Phys. Rev. Appl., 2017, 8, 044010.

31 Y. Sun, L. H. Vu, N. Chew, Z. Puthucheary, M. E. Cove and K. Zeng, ACS Biomater. Sci. Eng., 2019, 5, 478-486.

32 Z. Al-Rekabi and S. Contera, Proc. Natl. Acad. Sci. U. S. A., 2018, 115, 2658-2663.

33 W. Trewby, J. Faraudo and K. Voïtchovsky, Nanoscale, 2019, 11, 4376-4384.

34 M. E. F. Brollo, A. Domínguez-Bajo, A. Tabero, V. Domínguez-Arca, V. Gisbert, G. Prieto, C. Johansson, R. Garcia, A. Villanueva, M. C. Serrano and M. del P. Morales, ACS Appl. Mater. Interfaces, 2020, 12, 42954307.

35 E. N. Athanasopoulou, N. Nianias, Q. K. Ong and F. Stellacci, Nanoscale, 2018, 10, 23027-23036.

36 I. González-Domínguez, S. Gutiérrez-Granados, L. Cervera, F. Gòdia and N. Domingo, Biophys. J., 2016, 111, 11731179.

37 Y. Li, C. Yu, Y. Gan, P. Jiang, J. Yu, Y. Ou, D.-F. Zou, C. Huang, J. Wang, T. Jia, Q. Luo, X.-F. Yu, H. Zhao, C.-F. Gao and J. Li, npj Comput. Mater., 2018, 4, 49.

38 R. Giridharagopal, L. Q. Flagg, J. S. Harrison, M. E. Ziffer, J. Onorato, C. K. Luscombe and D. S. Ginger, Nat. Mater., 2017, 16, 737-742.

39 M. F. Dupont, A. Elbourne, E. Mayes and K. Latham, Phys. Chem. Chem. Phys., 2019, 21, 20219-20224.

40 E. T. Herruzo, H. Asakawa, T. Fukuma and R. Garcia, Nanoscale, 2013, 5, 2678-2685.

41 H. K. Nguyen, M. Ito and K. Nakajima, Jpn. J. Appl. Phys., 2016, 55, 08NB06.

42 A. Bubendorf, S. Walheim, T. Schimmel and E. Meyer, Beilstein J. Nanotechnol., 2018, 9, 1-10.
43 R. W. Stark, N. Naujoks and A. Stemmer, Nanotechnology, 2007, 18, 065502.

44 U. Bostanci, M. K. Abak, O. Aktaş and A. Dâna, Appl. Phys. Lett., 2008, 92, 093108.

45 X. D. Ding, J. An, J. B. Xu, C. Li and R. Y. Zeng, Appl. Phys. Lett. , 2009, 94, 223109.

46 H. T. Thompson, F. Barroso-Bujans, J. G. Herrero, R. Reifenberger and A. Raman, Nanotechnology, 2013, 24, 135701.

47 S. Kawai, F. F. Canova, T. Glatzel, T. Hynninen, E. Meyer and A. S. Foster, Phys. Rev. Lett., 2012, 109, 146101.

48 N. Liu, R. Dittmer, R. W. Stark and C. Dietz, Nanoscale, 2015, 7, 11787-11796.

49 J. L. Garrett, M. S. Leite and J. N. Munday, ACS Appl. Mater. Interfaces, 2018, 10, 28850-28859.

50 J. W. Li, J. P. Cleveland and R. Proksch, Appl. Phys. Lett., 2009, 94, 163118.

51 C. Dietz, E. T. Herruzo, J. R. Lozano and R. Garcia, Nanotechnology, 2011, 22, 125708.

52 J. Schwenk, M. Marioni, S. Romer, N. R. Joshi and H. J. Hug, Appl. Phys. Lett., 2014, 104, 112412.

53 J. Schwenk, X. Zhao, M. Bacani, M. A. Marioni, S. Romer and H. J. Hug, Appl. Phys. Lett., 2015, 107, 132407.

54 L. Stühn, J. Auernhammer and C. Dietz, Sci. Rep., 2019, 9, 17755.

55 H. J. Hug, B. Stiefel, P. J. A. Van Schendel, A. Moser, R. Hofer, S. Martin, H. J. Güntherodt, S. Porthun, L. Abelmann, J. C. Lodder, G. Bochi and R. C. O'Handley, J. Appl. Phys., 1998, 83, 5609-5620.

56 E. Meyer, H. J. Hug and R. Bennewitz, Scanning Probe Microscopy: Lab on a tip, Springer-Verlag, Berlin, 2004.

57 U. Hartmann, Annu. Rev. Mater. Sci., 1999, 29, 53-87.

58 A. Schwarz and R. Wiesendanger, Nano Today, 2008, 3, 28-39.

59 Y. Martin and H. K. Wickramasinghe, Appl. Phys. Lett., 1987, 50, 1455-1457.

60 J. J. Sáenz, N. Garcia, P. Grütter, E. Meyer, H. Heinzelmann, R. Wiesendanger, L. Rosenthaler, H. R. Hidber and H. J. Güntherodt, J. Appl. Phys., 1987, 62, 4293-4295.

61 A. San Paulo and R. Garcia, Phys. Rev. B: Condens. Matter Mater. Phys., 2001, 64, 193411.

62 T. R. Rodriguez and R. Garcia, Appl. Phys. Lett., 2002, 80, 1646-1648.

63 J. R. Lozano and R. Garcia, Phys. Rev. Lett., 2008, 100, 076102.

64 S. Kawai, T. Glatzel, S. Koch, B. Such, A. Baratoff and E. Meyer, Phys. Rev. Lett., 2009, 103, 220801.

65 S. Hosaka, A. Kikukawa, Y. Honda, H. Koyanagi and S. Tanaka, Jpn. J. Appl. Phys., 1992, 31, L904-L907.

66 M. Jaafar, O. Iglesias-Freire, L. Serrano-Ramón, M. R. Ibarra, J. M. de Teresa and A. Asenjo, Beilstein J. Nanotechnol., 2011, 2, 552-560.

67 X. Zhao, J. Schwenk, A. O. Mandru, M. Penedo, M. Baćani, M. A. Marioni and H. J. Hug, New J. Phys., 2018, 20, 013018.

68 A. Asenjo, J. M. García, D. García, A. Hernando, M. Vázquez, P. A. Caro, D. Ravelosona, A. Cebollada and F. Briones, J. Magn. Magn. Mater., 1999, 196-197, 23-25. 
69 J. R. Lozano, D. Kiracofe, J. Melcher, R. Garcia and A. Raman, Nanotechnology, 2010, 21, 465502.

70 J. E. Sader, R. Borgani, C. T. Gibson, D. B. Haviland, M. J. Higgins, J. I. Kilpatrick, J. Lu, P. Mulvaney,
C. J. Shearer, A. D. Slattery, P.-A. Thorén, J. Tran, H. Zhang, H. Zhang and T. Zheng, Rev. Sci. Instrum., 2016, 87, 093711.

71 A. Labuda, M. Kocun, M. Lysy, T. Walsh, J. Meinhold, T. Proksch, W. Meinhold, C. Anderson and R. Proksch, Rev. Sci. Instrum., 2016, 87, 073705. 